\title{
A Pediatric Patient with Idiopathic Short Stature Who Developed Obstructive Sleep Apnea after Starting Growth Hormone Replacement Therapy
}

Morkous SS $1,2,3 *$

${ }^{1}$ Department of Pediatrics, Pediatric Neurology Division, Lehigh Valley Children's Hospital, Allentown, PA, USA

${ }^{2}$ Pediatrics Core Academic Rank, Morsani College of Medicine, University of South Florida, Tampa, FL, USA

${ }^{3}$ Department of Clinical Sciences, DeSales University, Center Valley, PA, USA

\begin{abstract}
Background: Growth hormone (GH) therapy has long been suspected to induce obstructive sleep apnea (OSA) in children and adults. Moreover, reports about GH-associated sudden death in children with Prader-Willi syndrome (PWS) have prompted concerns about GH worsening sleep apnea. Previous studies have supported routine polysomnography for children with PWS prior to starting GH treatments, regardless of clinical history. However, there are no established guidelines recommending routine polysomnography (PSG) prior to the commencement of $\mathrm{GH}$ therapy in other pediatric patients.
\end{abstract}

Case description: We report a case of a 15-year-old young man with intractable headaches, referred to the sleep clinic to rule out any sleep-related variables. After an initial non-significant (mild snoring) sleep study, the patient returned with worsening snoring about one year after starting GH therapy for concerns of short stature.

Results: A second polysomnogram revealed that his obstructive apnea-hypopnea index had risen dramatically from baseline. His symptoms resolved after tonsillectomy and adenoidectomy.

Conclusion: This interesting case highlights the need for caution with any patient eligible for GH therapy. We recommend additional research to look in the development of definitive guidelines regarding the indications for polysomnography for patients with idiopathic short stature and non-significant initial sleep history-particularly before and during the administration of GH therapy.

Keywords: Growth hormone; Growth hormone therapy; Pediatrics; Obstructive sleep apnea; Prader-Willi syndrome; Idiopathic short stature

Abbreviations: A and T: Adenoidectomy and Tonsillectomy; ESS: Epworth Sleepiness Scale; GH Growth Hormone; OSA: Obstructive Sleep Apnea; PSG: Polysomnogram; PSQ: Pediatric Sleep Questionnaire; PWS: Prader Willi Syndrome

\section{Introduction}

Growth hormone (GH) therapy has long been suspected to induce obstructive sleep apnea (OSA) in children and adults $[1,2]$. Moreover, reports about $\mathrm{GH}$-associated sudden death in children with Prader-Willi syndrome (PWS), a genetic disorder associated with morbid obesity and a high prevalence of OSA, have prompted concerns about GH worsening sleep apnea [3]. These concerns have led to recommendations for PSG before and after starting GH therapy, as well as careful monitoring for sleep apnea in children with PWS regardless of the clinical history [3-5]. However, there are no established guidelines recommending routine polysomnography (PSG) prior to the commencement of GH therapy in pediatric patients with other medical conditions who do not have PWS.

We report a case of a pediatric patient with an initial non-significant sleep history and a sleep study and, who developed OSA one year after starting GH therapy.

\section{Case Report}

A 15-year-old young man presented to the Neurology and Sleep Clinic for the first time for an evaluation regarding intractable headaches that developed after a recent concussion. The referring provider ordered an evaluation to be done by the Pediatric Sleep Medicine specialist together with a plan for a pre-visit polysomnogram (PSG) to rule out sleep-related variables (e.g., sleep apnea and circadian rhythm sleep disorders) as potential causes for the headaches. After the PSG was done, the patient was subsequently seen in the Sleep Clinic. At that time, the child snored mildly. Epworth Sleepiness Scale (ESS) was $4 / 24$ with a regular sleep-wake cycle and no other significant sleep history.

Examination revealed Mallampati score of 1 and a tonsil size of 0 (absent) with no other physical exam concerns except for short stature. His neck circumference was 16 inches $(40.64 \mathrm{~cm})$. The initial PSG (Figure 1A) revealed a total sleep time of 460 minutes, obstructive apnea hypopnea index of 0.4 events/h, and an arousal index of $8.6 / \mathrm{h}$.

Nine months later, the patient was evaluated in the Endocrine Clinic for concerns related to his short stature and pubertal delay (Tanner stage 2: pubic hair with testicular volume $6 \mathrm{ml}$ bilaterally). The patient had no signs of other hypothalamic or pituitary hormone deficiencies such as hypogonadism, central adrenal insufficiency, or central hypothyroidism. MRI of the brain and pituitary gland with and without contrast was done at that time as part of his evaluation and was normal. Thus, he was diagnosed with idiopathic GH deficiency

*Corresponding author: Dr. Sameh S. Morkous, Department of Pediatrics, Children's Hospital at Lehigh Valley, 1210 S. Cedar Crest Blvd., Suite 2400 Allentown, PA-18103-6229, USA, Tel: 610-402-3888, Fax: 610-402-3894; E-mail: samehserry20@hotmail.com

Received October 30, 2017; Accepted November 25, 2017; Published November 28, 2017

Citation: Morkous SS (2017) A Pediatric Patient with Idiopathic Short Stature Who Developed Obstructive Sleep Apnea after Starting Growth Hormone Replacement Therapyl. J Mol Genet Med 11: 307 doi:10.4172/1747-0862.1000307

Copyright: (C) 2017 Morkous SS. This is an open-access article distributed under the terms of the Creative Commons Attribution License, which permits unrestricted use, distribution, and reproduction in any medium, provided the original author and source are credited 

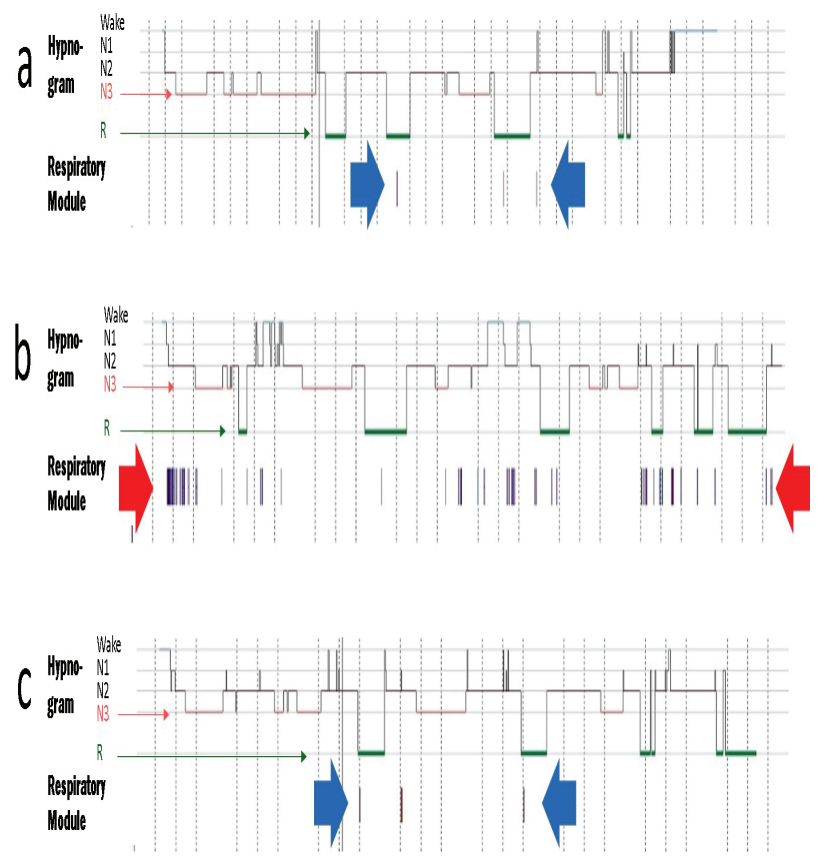

Figure 1: (A-C): Comparison of percentage of total sleep time in each stage for baseline PSG and the 2 follow-up PSGs. Normal mean sleep stage percentages for ages $13-16$ y are: N1, mean $4 \%$ (range 0-9), N2, mean $43 \%$ (range 25-57), N3, mean 32\% (range 17-48), R, mean 22\% (range 16-30) [9].

\begin{tabular}{|c|c|c|c|c|}
\hline Characteristics & Initial baseline (pre-GH therapy) & Post-GH therapy & Change $^{a}$ & Post-A\&T \\
\hline Weight, percentile ${ }^{b}$ & $63.96 \mathrm{~kg}, 79^{\text {th }}$ & $67.6 \mathrm{~kg}, 75^{\text {th }}$ & -0.10 & $69.9 \mathrm{~kg}, 76^{\text {th }}$ \\
\hline Height, percentile ${ }^{b}$ & $151.5 \mathrm{~cm}, 2^{\text {nd }}$ & $158.5 \mathrm{~cm}, 4^{\text {th }}$ & +0.26 & $161.0 \mathrm{~cm}, 5^{\text {th }}$ \\
\hline BMI, percentile & $27.87 \mathrm{~kg} / \mathrm{m}^{2}, 96^{\text {th }}$ & $26.9 \mathrm{~kg} / \mathrm{m}^{2}, 94^{\text {th }}$ & & $27.0 \mathrm{~kg} / \mathrm{m}^{2}, 94^{\text {th }}$ \\
\hline BMI z-score & 1.81 & 1.58 & -0.23 & 1.54 \\
\hline Neck circumference & $40.64 \mathrm{~cm}$ & $41.7 \mathrm{~cm}$ & $+1.34 \mathrm{~cm}(+2.6 \%)$ & -- \\
\hline \multicolumn{5}{|c|}{ Sleep characteristics } \\
\hline Lights off/on & 9:30 pm/6:00 am & $10: 00$ pm/6:22 am & & $10: 15 \mathrm{pm} / 6: 18 \mathrm{am}$ \\
\hline Total sleep time & $460 \min$ & $421.5 \mathrm{~min}$ & & $425.9 \min$ \\
\hline Total supine time & $460 \min (100 \%)$ & $6.5 \min (0.01 \%)$ & & $196 \min (46 \%)$ \\
\hline Sleep efficiency & $90.1 \%$ & $92.3 \%$ & & $97.3 \%$ \\
\hline \multicolumn{5}{|c|}{ Apnea-hypopnea index (events/h) } \\
\hline Obstructive & 0.4 & 8.4 & +8 & 0.1 \\
\hline REM & 1.5 & 4.4 & +2.9 & 0 \\
\hline Obstructive Supine & 0.4 & 9.4 & +9 & 0 \\
\hline Arousal index & $8.6 / \mathrm{h}$ & $14.6 / \mathrm{h}$ & & $6.1 / \mathrm{h}$ \\
\hline Sleep latency & $16 \min$ & $13 \mathrm{~min}$ & & $7.5 \mathrm{~min}$ \\
\hline \multicolumn{5}{|c|}{ Lowest $\mathrm{O}_{2}$ saturation } \\
\hline NREM & $93 \%$ & $92 \%$ & & $94 \%$ \\
\hline REM & $92 \%$ & $89 \%$ & & $93 \%$ \\
\hline Mean $\mathrm{EtCO}_{2}$ & $36 \mathrm{mmHg}$ & $40 \mathrm{mmHg}$ & & $45.8 \mathrm{mmHg}$ \\
\hline Periodic limb movement index & 0.0 events $/ \mathrm{h}$ & 0.0 events/h & & 0.0 events $/ \mathrm{h}$ \\
\hline $\begin{array}{l}\text { Note: A\&T: Adenoidectomy and Ton } \\
\text { a Where relevant, change from base } \\
\text { b Based on CDC 2-20 years weight- }\end{array}$ & $\begin{array}{l}\text { ny; GH: Growth Hormone; PSG: } \\
\text { ost-GH therapy has been calcula } \\
\text { and years stature-for-age data. }\end{array}$ & gram. & & \\
\hline
\end{tabular}

Table 1: Comparison of patient characteristics and PSG results: At baseline, post-GH therapy (1 y after baseline) and post A\&T ( 1.5 y after baseline).

and subsequently started on somatropin growth hormone, $2.5 \mathrm{mg}$ daily subcutaneously. Within 4 months, he presented again to the Sleep Clinic with concerns raised primarily by the family for worsening snoring and their new observation of sleep respiratory pauses (ESS had risen slightly to 5/24). On physical exam, his tonsil size was now $2+$ (extends to beyond the pillars) and Mallampati score was 2. His neck circumference was now 16.4 inches $(41.7 \mathrm{~cm})$.
These new concerns prompted the decision to repeat the PSG (Figure 1B), which revealed that his obstructive apnea-hypopnea index had risen dramatically from baseline (Table 1). The patient was then diagnosed with mild to moderate OSA and referred to an Ear, Nose and Throat specialist for tonsillectomy and adenoidectomy, after which his sleep apnea resolved, as evidenced on the third PSG done 3 months after the surgery (Figure 1C and Table 1). With resolution of the sleep 
$60.0 \%$

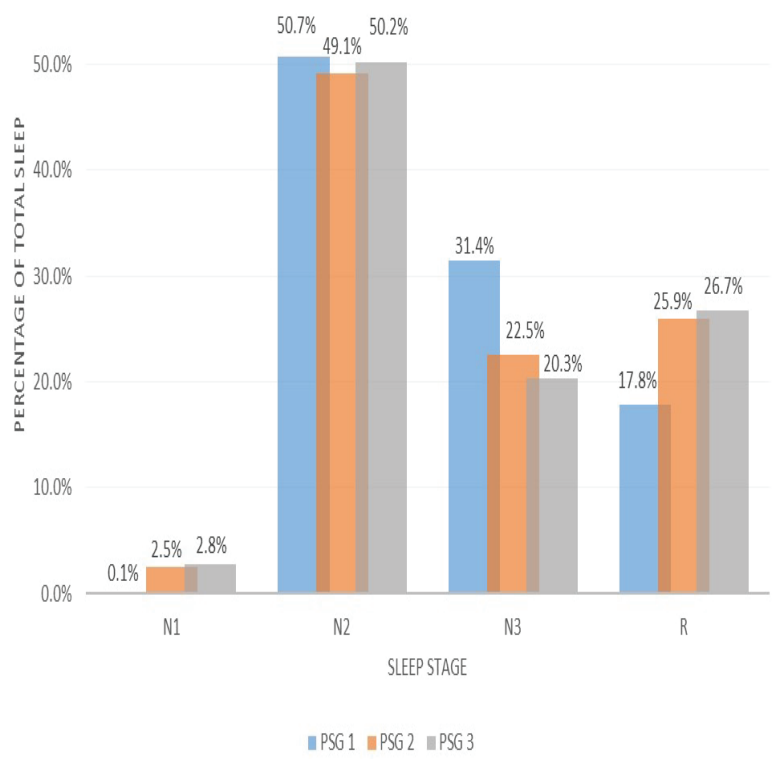

Figure 2: Hypnograms from the patient's three PSGs: (a) baseline, (b) post-GH therapy, and (c) follow-up after T\&A. Large red arrows in $1 \mathrm{~b}$ point to the large increase in obstructive respiratory events, when compared with those in $1 \mathrm{a}$ and $1 \mathrm{c}$, indicated by the blue arrows. Narrow red and green arrows indicate stages N3 and R sleep, respectively.

apnea, and benefits outweighing the risks for this patient, GH therapy was continued.

It is noteworthy to mention that the headaches questioned by the referring provider initially in this case were most probably related to the history of the underlying concussions, a conclusion based on the patient's medical history and the initial PSG findings. His headaches improved in the follow-up visits.

\section{Discussion}

In general, there is a paucity of published literature on the sleep characteristics of children with GH deficiency. A recent study by Verillo and colleagues [6] demonstrated significant sleep architecture changes - with relatively more sleep time in both stages N1 and N3 and relatively less time in stage $\mathrm{R}$-for $\mathrm{GH}$ deficient children as compared with age-matched normal children. Confirming previous reports [7-9], they noted a significant decrease in stage N3 after GH therapy [6]. The patient in this case had comparable findings in stage $\mathrm{N} 3$, with a baseline of 144.5 minutes (31.4\%). After starting GH therapy, this time was subsequently reduced to 95 minutes $(22.5 \%,-\Delta 49.5 \mathrm{~min})$ in stage $\mathrm{N} 3$. In general, a normal adolescent's slow-wave sleep would be expected to initially decline-from Tanner stage 1 (age 10-12 years) to Tanner stage 5 (age 14-16 years)—by approximately 40\% [10,11]. However, these expected relatively lower values for age were not evident in our patient's initial PSG. After the surgery, the patient's third PSG shows the percentage of time in N3 as remaining relatively stable at $20.3 \%$ and thus relatively similar to the second PSG (Figure 2).

Similar to what was demonstrated in the Verillo study [6], a relatively lower initial sleep time in stage $\mathrm{R}$ on the first study was also noted for this patient as compared with age-matched normal children. He had a baseline of 82 minutes (17.8\%), which subsequently increased to 109 minutes $(25.9 \%,+\Delta 27 \mathrm{~min})$ on the second study and remained relatively stable at $26.7 \%$ after his surgery on the third study (Figure 2).
The worsening of his snoring and the newly reported sleep respiratory pauses that were observed by the family prompted the repeat (second) PSG, in which the most dramatic change, post-hormone therapy, was the increase in the obstructive events (Figure 1D) and with a very minimal and clinically non-significant change in the central events (Table 1). Possible causes for this newly developed OSA could include weight gain. However, this patient had actually reduced his BMI $z$-score during the one-year interval between the initial (normal) and the first follow-up (abnormal) PSG (Table 1).

No other risk factors (e.g., family history or seasonal allergies or dysmorphic features or recurrent tonsillar infections) were identified. The patient spent a drastically reduced amount of supine time in the second study, also had similar lights off/on times in the three PSGs, and the increased REM sleep time was observed on both the second abnormal and the third non-significant repeat PSGs (Table 1). These findings argue against any other alternative plausible risk factors contributing to the newly developed OSA on the second study. This together with the absence of any other identified risk factors for OSA in this patient, the reduced BMI z-score during the one-year interval, the observed changes in the sleep history and also the physical exam findings over that time period in addition to the current three PSGs findings strongly point to $\mathrm{GH}$ as the culprit which has been the only major factor that changed over time for this patient.

Mechanism of growth hormone-induced OSA in this patient would include tonsillar and adenoidal hypertrophy [1]. Additional possible mechanisms might be related to the slight but measurable increase in neck circumference $(+1.06 \mathrm{~cm})$ noticed on the second exam, which has been previously described in the literature as a potential culprit [12]. It has also been postulated that $\mathrm{GH}$ and insulin-like growth factor I may have a direct role in the pathogenesis of sleep apnea through macroglossia and pharyngeal swelling, especially in obese patients [13]. The occurrence of OSA is associated with a reduction in IGF-1 levels [14]; however, these observations are controversial.

Syndromes with hereditary GH deficiency (e.g., PWS) are often associated with obesity along with craniofacial and pharyngeal abnormalities predisposing to OSA. A small number of published studies have provided limited data in regard to the potential clinical utility for PSGs in children with PWS who are receiving or being considered for GH supplementation. Wise et al. [15] recently reported that there is no sufficient concrete support for the routine use of PSG to predict risk of death or to monitor for development of significant cardiorespiratory abnormalities in this population. Conversely, there are other studies that support the routine PSG in children with PWS [3-5].

\section{Conclusion}

In this case, a child with an idiopathic short stature and a benign initial sleep history as well as a non-significant initial PSG developed OSA about one year after starting GH therapy, at which time a repeat PSG was triggered by the family's new observations. This case illustrates the main characteristic sleep architecture changes observed with GH deficiency and GH therapy that are described in the literature. It also highlights the importance of screening patients with idiopathic short stature who are eligible for GH use for symptoms of sleep apnea and identifying a possible need for PSG either before or during GH therapy regardless of the initial sleep history. The observations noticed in this case raise the attention to a potentially important and possibly an underdiagnosed problem. Additional studies with a larger number of subjects and longitudinal data incorporating the use of surveys (e.g., the Pediatric Sleep Questionnaire) in patients with idiopathic short stature 
Citation: Morkous SS (2017) A Pediatric Patient with Idiopathic Short Stature Who Developed Obstructive Sleep Apnea after Starting Growth Hormone Replacement Therapyl. J Mol Genet Med 11: 307 doi:10.4172/1747-0862.1000307

who are eligible for GH therapy are needed to replicate the interesting findings in this case and to develop a physical characteristic profile to identify specific risk factors for OSA and/or sudden death with the general use of GH in this population. This information can be used to establish general concrete guidelines for screening patients for sleep apnea prior to or during GH therapy in this population.

\section{Conflict of Interest}

The author declares no conflict of interest.

\section{Statement of Ethics}

As this is just a description of a single case and the patient is not identifiable our organization's institutional review board does not require board approval.

\section{Acknowledgement}

The author is grateful to Jacqueline Grove for manuscript editing and formatting. This work was supported by National Institutes of Health with gran number 4848623124

\section{References}

1. Gerard JM, Garibaldi L, Myers SE, Aceto T Jr, Kotagal S, et al. (1997) Sleep apnea in patients receiving growth hormone. Clin Pediatr (Phila) 36: 321-326.

2. Nolte W, Radisch C, Rodenbeck A, Wiltfang J, Hüfner M (2002) Polysomnographic findings in five adult patients with pituitary insufficiency before and after cessation of human growth hormone replacement therapy. Clin Endocrinol 56: 805-810.

3. Miller J, Silverstein J, Shuster J, Driscoll DJ, Wagner M (2006) Short-term effects of growth hormone on sleep abnormalities in Prader-Willi syndrome. $J$ Clin Endocrinol Metab 91: 413-417.

4. Vandeleur M, Davey MJ, Nixon GM (2013) Are sleep studies helpful in children with Prader-Willi syndrome prior to commencement of growth hormone therapy? J Paediatr Child Health 49: 238-241.
5. Deal CL, Tony M, Hoybye C Allen DB, Tauber M, et al. (2013) Growth hormone in Prader-Willi syndrome clinical care guidelines workshop for the participants: Growth hormone research society workshop summary: Consensus guidelines for recombinant human growth hormone therapy in Prader-Willi syndrome. J Clin Endocrinol Metab 98: e1072-e1087.

6. Verillo E, Bizzarri C, Bruni O, Ferri R, Parone M, et al. (2012) Effects of replacement therapy on sleep architecture in children with growth hormone deficiency. Sleep Med 13: 496-502.

7. Wu RH, Thorpy MJ (1988) Effect of growth hormone treatment on sleep EEGs in growth hormone-deficient children. Sleep 11: 425-429.

8. Mendelson WB, Slater S, Gold P, Gillin JC (1980) The effect of growth hormone administration on human sleep: a dose-response study. Biol Psychiatry 15 613-618.

9. Van den Bossche RAS, De Weerd AW (2007) Normal values of polysomnography in children. Sleep Wake Res Netherlands 18: 37-40.

10. Marcus CL, Carroll JL, Donnelly DF, Loughlin GM (2008) Sleep in children: Developmental changes in sleep patterns. ( $2^{\text {nd }}$ edn). Boca Raton, Florida, USA

11. Carskadon MA (2011) Sleep in adolescents: The perfect storm. Pediatr Clin North Am 58: 637-647.

12. Karimi M, Koranyi J, Franco C, Peker Y, Eder DN, et al. (2010) Increased neck soft tissue mass and worsening of obstructive sleep apnea after growth hormone treatment in men with abdominal obesity. J Clin Sleep Med 6: 256-263.

13. Gomes C, Pereira C, Ferreira R (2013) Sleep disordered breathing and growth hormone therapy in children with Prader Willi syndrome. Sleep Med 14: e141.

14. Izumi S, Fernando F, Ribeiro-Filho, Carneiro G, Sônia M, et al. (2016) IGF-1 levels are inversely associated with metabolic syndrome in obstructive sleep apnea. J Clin Sleep Med 12: 487-493.

15. Wise MS, Nichols CD, Grigg-Damberger MM (2011) Executive summary of respiratory indications for polysomnography in children: An evidence-based review. Sleep 34: 389-398 